TITLE:

\title{
One-dimensional self-gravitating sheet model and Lynden-Bell statistics.
}

\author{
$\operatorname{AUTHOR}(\mathrm{S})$ :
}

Yamaguchi, Yoshiyuki Y

\section{CITATION:}

Yamaguchi, Yoshiyuki Y. One-dimensional self-gravitating sheet model and Lynden-Bell statistics.. Physical review. E, Statistical, nonlinear, and soft matter physics 2008, 78 : 041114.

ISSUE DATE:

2008-10-13

URL:

http://hdl.handle.net/2433/202514

RIGHT:

(C)2008 The American Physical Society 


\title{
One-dimensional self-gravitating sheet model and Lynden-Bell statistics
}

\author{
Yoshiyuki Y. Yamaguchi* \\ Department of Applied Mathematics and Physics, Kyoto University, Kyoto, 606-8501, Japan
}

(Received 23 June 2008; published 13 October 2008)

\begin{abstract}
The Lynden-Bell statistics has been proposed to explain common features among galaxies, which are not in thermal equilibrium. The statistics is not successful to reproduce energy distribution in the one-dimensional self-gravitating sheet model except for initial states near the virial equilibrium. The breakdown is caused by dynamically accelerated high-energy sheets, and hence a modified statistics is examined by focusing on lowenergy sheets in order to clarify validity of the basic idea of the Lynden-Bell statistics. The modification improves agreement between the theoretical and numerical energy distributions in a wide interval of the initial virial ratio.
\end{abstract}

DOI: 10.1103/PhysRevE.78.041114

PACS number(s): 05.20.- $\mathrm{y}, 05.45 .-\mathrm{a}$

\section{INTRODUCTION}

In a galaxy, the two-body relaxation time is typically of order $10^{17} \mathrm{yr}$, and is much longer than the life time of the universe, which is of order $10^{10} \mathrm{yr}$. The galaxy is hence not in the thermal equilibrium, but common features are observed in galaxies, for instance, brightness profiles in elliptical galaxies. To explain the common features, a nonequilibrium statistical mechanics is expected.

One of such statistical mechanics has been proposed by Lynden-Bell [1]. Collisions between stars can be neglected in a short time region comparing with the two-body relaxation time, and hence temporal evolution of a galaxy is approximately governed by the Vlasov equation. The Vlasov equation preserves area elements in $\mu$ space, and the statistical mechanics is based on exclusivity between the area elements. Entropy led by the exclusivity is maximized by distribution of Fermi-Dirac type. It is worth noting that the distribution depends on initial states, while equilibrium distribution does not, and the distribution is completely determined by the initial state. The initial distribution must be a stepwise function on $\mu$ space.

The Lynden-Bell statistics has been mainly examined in the one-dimensional self-gravitating sheet model. We refer to this model as the sheet model. Advantages of the sheet model are that no evaporation occurs due to compactness of an energy surface and that temporal evolution is exactly discretized for accurate numerical computations. In the beginning agreement between the Lynden-Bell statistics and $N$-body simulations has been reported in the sheet model for two-level [2] and three-level [3] initial distributions. However, the agreement tends to break as initial virial ratio becomes large. Disagreement is observed both in the $N$-body simulations [4,5], and in simulations of the Vlasov equation [6]. After many reports on disagreements, the Lynden-Bell statistics is regarded as an inapplicable theory to selfgravitating systems.

Nevertheless, similar statistical mechanics have been developed for Hamiltonian systems having long-range interactions. Such a system is frequently trapped at a long-lasting quasistationary state (QSS) before relaxing towards thermal equilibrium. QSSs are numerically observed in the sheet model [7], in free electron lasers [8], and in the Hamiltonian mean-field model [9]. In magnetized pure-electron plasma, QSSs are observed numerically [10,11] and experimentally [12-14]. Relaxation from an initial state to QSS is approximately described by the Vlasov equation $[15,16]$, and the Fermi-Dirac-type distribution based on the exclusivity successfully explain distribution in QSS in the free electron lasers [8] and in the Hamiltonian mean-field model $[17,18]$. The exclusivity is also useful in the two-dimensional inviscid incompressible fluid [19,20], and in pure electron plasmas [21]. Analogy between the two statistical treatments for the collisionless self-gravitating systems and for the twodimensional Euler fluids is discussed in Ref. [22]. The Lynden-Bell statistics is expected to catch a part of statistical properties even in the self-gravitating systems.

In the sheet model, sheets are classified into core and halo, which are sets of low- and high-energy sheets, respectively. Halo is generated by dynamical acceleration of the sheets [4], and energy distribution has several peaks in a high-energy interval, which never appear in the Fermi-Diractype distribution. Due to the dynamical acceleration halo does not obey the entropy maximum principle, and hence a statistical theory possibly breaks. On the other hand, density of sheets is high enough in core as the exclusivity must be effective, and a core may be formed by the entropy maximum principle. The main purpose of this paper is to examine the usefulness of the exclusivity in the sheet model. Distributions derived from a modified Lynden-Bell statistics fit numerically obtained ones if we focus on the core only.

This paper is constructed as follows. The sheet model and the associated Vlasov equation are introduced in Sec. II, and a family of initial distributions is shown in Sec. III. The Lynden-Bell distribution for the whole system and for core are derived in Secs. IV and V, respectively. Section VI is devoted to summary and discussions.

\section{MODEL}

The sheet model is represented by the Hamiltonian 


$$
\mathcal{H}(X, P)=\sum_{j=1}^{N} \frac{P_{j}^{2}}{2 m_{j}}+2 \pi G \sum_{j<k}^{N} m_{j} m_{k}\left|X_{j}-X_{k}\right|,
$$

where $X_{j}$ and $P_{j}$ are the position and its conjugate momentum of the $j$ th sheet, respectively, and $G$ is the gravitational constant. We use the simple expressions which are similar to $X=\left(X_{1}, \ldots, X_{N}\right)$ and $P=\left(P_{1}, \ldots, P_{N}\right)$ in this paragraph. For simplicity we assume that each sheet has an identical mass, i.e., $m_{j}=m$. To recover the extensivity of the system, we perform the canonical transformation

$$
X=\frac{x}{a}, \quad P=a p, \quad a=(2 \pi G N)^{1 / 3} m
$$

and the transformed Hamiltonian is

$$
H(x, p)=\frac{a^{2}}{m}\left[\sum_{j=1}^{N} \frac{p_{j}^{2}}{2}+\frac{1}{N} \sum_{j<k}^{N}\left|x_{j}-x_{k}\right|\right] .
$$

The overall factor $a^{2} / m$ can be neglected by changing time scale, and hence we consider the following simple Hamiltonian system:

$$
H(x, p)=\sum_{j=1}^{N} \frac{p_{j}^{2}}{2}+\frac{1}{N} \sum_{j<k}^{N}\left|x_{j}-x_{k}\right| .
$$

The factor $1 / N$ in the potential term makes the system extensive and hence specific energy $U$, the energy per one particle, is finite in the limit $N \rightarrow \infty$.

Later $N$-body simulations are performed by using an exact discretization code. Each sheet freely falls to the center of mass unless collisions happen, and hence canonical equations of motion for the sheet model (4) are exactly solved in a time region between two consecutive collisions. We can therefore discretize the canonical equations of motion as a mapping from a collision to the next, without the aid of any integration schemas.

The discrete $N$-body system (4) is approximated by the associated Vlasov equation

$$
\frac{\partial f}{\partial t}+\frac{\partial H_{1}}{\partial p} \frac{\partial f}{\partial x}-\frac{\partial H_{1}}{\partial x} \frac{\partial f}{\partial p}=0
$$

where $x, p \in \mathbb{R}$, the one-body Hamiltonian $H_{1}(x, p, t)$ is defined as

$$
H_{1}(x, p, t)=\frac{p^{2}}{2}+\Phi[f](x, t)
$$

and the one-body potential $\Phi[f](x, t)$ is

$$
\Phi[f](x, t)=\iint_{\mathbb{R}^{2}}|x-y| f(y, p, t) d y d p .
$$

The Vlasov equation (5) conserves the following three quantities:

$$
1=\iint_{\mathbb{R}^{2}} f(x, p, t) d x d p
$$

$$
\begin{gathered}
U=\iint_{\mathbb{R}^{2}}\left(\frac{p^{2}}{2}+\frac{\Phi[f](x, t)}{2}\right) f(x, p, t) d x d p, \\
P=\iint_{\mathbb{R}^{2}} p f(x, p, t) d x d p .
\end{gathered}
$$

The first quantity is the normalization condition for $f$, and the second and the third represent conservations of specific energy and specific momentum, respectively. It is straightforward to show that the above three quantities are constants of time by using the Vlasov equation (5) and integration by parts with the assumption that $f$ rapidly goes to zero in the limits $|x| \rightarrow \infty$ and $|p| \rightarrow \infty$. Note that the integrand of Eq. (8b) differs from the one-body Hamiltonian (6), since the onebody potential $\Phi$ explicitly depends on time through distribution $f$.

The three quantities are constants of time, and hence their values are determined by a given initial condition. In Sec. IV, maximization of an entropy will be subject to these constraints.

\section{INITIAL DISTRIBUTION}

We consider a family of waterbag initial distributions on $\mu$ space, since it is the simplest type to which the LyndenBell statistics is applicable. The waterbag distribution is a two-level function expressed as

$$
f(x, p, 0)= \begin{cases}f_{0} & {\left[(x, p) \in D \subset \mathrm{R}^{2}\right],} \\ 0 & \text { (otherwise) } .\end{cases}
$$

We limit $D$ in rectangles such that

$$
D=\left\{(x, p) \in \mathbb{R}^{2}|| x|<\Delta x,| p \mid<\Delta p\right\} .
$$

The initial density $f_{0}$ is determined from the normalization condition (8a) as

$$
f_{0}=\frac{1}{|D|}=\frac{1}{4 \Delta x \Delta p},
$$

where $|D|$ represents the area of $D$.

For this family of initial distributions, initial values of the one-body potential $\Phi$ and energy $U$ are computed as

$$
\Phi[f](x, 0)= \begin{cases}\frac{x^{2}+(\Delta x)^{2}}{2 \Delta x} & (|x| \leqslant \Delta x), \\ |x| & (|x|>\Delta x)\end{cases}
$$

and

$$
U=\frac{(\Delta p)^{2}}{6}+\frac{\Delta x}{3},
$$

respectively. From the symmetry with respect to $p$, the specific momentum takes zero, i.e., $P=0$.

The family of initial distributions has two parameters, the pair of $\Delta x$ and $\Delta p$ or equivalently the pair of $f_{0}$ and $U$. However, the number of parameters is reduced to 1 thanks to invariance of canonical equations of motion of the system (4) and the Vlasov equation (5) under the scaling 


$$
x_{j} \mapsto c x_{j}, \quad p_{j} \mapsto \sqrt{c} p_{j}, \quad t \mapsto \sqrt{c} t(c>0) .
$$

The initial virial ratio, which is defined by

$$
r=\frac{2 K(0)}{V(0)}=\frac{(\Delta p)^{2}}{\Delta x}
$$

is invariant under the scaling (14) and hence is suitable to parametrize the family of initial distributions. Here $K(0)$ and $V(0)$ represent initial kinetic and potential energy, respectively. The specific energy $U$ is irrelevant, and we set $U=1$ without loss of generality.

\section{LYNDEN-BELL DISTRIBUTION FOR THE WHOLE}

The Lynden-Bell distribution is obtained by maximizing the entropy

$$
S[f]=-f_{0} \iint_{\mathbb{R}^{2}}\left[\frac{f}{f_{0}} \ln \frac{f}{f_{0}}+\left(1-\frac{f}{f_{0}}\right) \ln \left(1-\frac{f}{f_{0}}\right)\right] d x d p,
$$

with the three constraints (8). Here $f$ is a coarse-grained distribution, and the second term of the right-hand side comes from the exclusivity of area elements [1].

Introducing three Lagrange multipliers $\alpha, \beta$, and $\lambda$, which correspond to the constraints $(8 \mathrm{a})-(8 \mathrm{c})$, respectively, the variational problem of the entropy (16) is solved by the distribution

$$
f(x, p, t)=\frac{f_{0}}{e^{\alpha+\beta H_{1}(x, p, t)+\lambda p}+1} .
$$

Values of the three Lagrange multipliers are determined by substituting the distribution (17) into the three constraints (8). The vanishing specific momentum $P=0$, implies $\lambda=0$ and the distribution is hence simplified as

$$
f(x, p)=\frac{f_{0}}{e^{\alpha+\beta H_{1}(x, p)}+1} .
$$

We omitted the argument $t$ in $f$ and $H_{1}$, since the distribution (18) is a stationary solution to the Vlasov equation (5). The remaining two multipliers $\alpha$ and $\beta$ are determined by the two constraints (8a) and (8b), which are rewritten as follows.

Let $\Phi[f](x)$ have the symmetry $\Phi[f](-x)=\Phi[f](x)$, and $f(x, p)$ have the same symmetry accordingly. The symmetry implies that the one-body potential $\Phi[f](x)$ is an increasing function in $x>0$, since

$$
\begin{aligned}
\frac{d \Phi}{d x}(x) & =\iint_{\mathbb{R}^{2}} \frac{x-y}{|x-y|} f(y, p) d y d p \\
& =\int_{\mathbb{R}} d p\left(\int_{-\infty}^{-x}+\int_{-x}^{x}+\int_{x}^{\infty}\right) \frac{x-y}{|x-y|} f(y, p) d y \\
& =\int_{\mathbb{R}} d p \int_{-x}^{x} f(y, p) d y \geqslant 0 .
\end{aligned}
$$

The distribution $f(x, p)$ also has the symmetry with respect to $p, f(x,-p)=f(x, p)$, and hence the domain of integration in
Eqs. (8a) and (8b) is reduced from $\mathbb{R}^{2}$ to $[0, \infty) \times[0, \infty)$. To this end, the transformations $\phi=\Phi(x)$ in $x>0$ and $k$ $=\beta p^{2} / 2$ in $p>0$ are validated, and the two constraints become

$$
\begin{gathered}
1=4\left(\frac{f_{0}^{2}}{2 \beta^{3}}\right)^{1 / 4}\left[F_{1 / 2}(\nu)\right]^{1 / 2}, \\
U=\frac{1}{2 \beta}\left(\frac{f_{0}^{2}}{2 \beta^{3}}\right)^{1 / 4}\left[2 I_{K}(\nu)+I_{V}(\nu)\right]+\frac{1}{2 \beta} I_{\phi}(\nu) .
\end{gathered}
$$

The first term of Eq. (20b) represents kinetic energy, and the sum of the second and the third terms is potential energy. Here $I_{K}(\nu), I_{V}(\nu)$, and $I_{\phi}(\nu)$ are defined as

$$
\begin{aligned}
& I_{K}(\nu)=\int_{0}^{\infty} \frac{F_{1 / 2}(\nu-\zeta)}{\left[F_{1 / 2}(\nu)-F_{1 / 2}(\nu-\zeta)\right]^{1 / 2}} d \zeta, \\
& I_{V}(\nu)=\int_{0}^{\infty} \frac{\zeta F_{-1 / 2}(\nu-\zeta)}{\left[F_{1 / 2}(\nu)-F_{1 / 2}(\nu-\zeta)\right]^{1 / 2}} d \zeta,
\end{aligned}
$$

and

$$
I_{\phi}(\nu)=\int_{0}^{\infty}\left[\left(\frac{F_{1 / 2}(\nu)}{F_{1 / 2}(\nu)-F_{1 / 2}(\nu-\zeta)}\right)^{1 / 2}-1\right] d \zeta,
$$

respectively, and $F_{n}(x)$ is the Fermi-Dirac integral defined as

$$
F_{n}(x)=\int_{0}^{\infty} \frac{k^{n}}{e^{k-x}+1} d k .
$$

The new variables $\nu$ and $\zeta$ are defined as

$$
\nu=-\alpha-\beta \phi_{0}, \quad \zeta=\beta\left(\phi-\phi_{0}\right),
$$

respectively, and the zero-point potential energy $\phi_{0}$ $=\Phi[f](0)$ is expressed as

$$
\phi_{0}=\frac{1}{\beta} I_{\phi}(\nu) .
$$

We used the relation for the Fermi-Dirac integral

$$
\frac{d}{d x} F_{n}(x)=n F_{n-1}(x)(n>0)
$$

to derive Eqs. (20a) and (26).

The normalization condition (20a) solves $\beta$ as a function of $\nu$ as

$$
\beta=\left\{2^{7}\left[f_{0} F_{1 / 2}(\nu)\right]^{2}\right\}^{1 / 3}
$$

and, substituting this expression of $\beta$ into Eq. (20b), we obtain an equation for $\nu$. A solution $\nu$ to the equation determines $\beta$ by Eq. (28), $\phi_{0}$ by Eq. (26) and $\alpha$ by $\alpha=-\nu-\beta \phi_{0}$.

We observe energy distribution instead of distribution on $\mu$ space itself. Energy $\epsilon$ represents the value of the one-body Hamiltonian $H_{1}(x, p)$ and energy density $D(\epsilon)$ is computed as [5] 


$$
D(\epsilon)=\left(\frac{\beta}{2 f_{0}^{2}}\right)^{1 / 4} \int_{0}^{\beta\left(\epsilon-\phi_{0}\right)} \frac{\left[\beta\left(\epsilon-\phi_{0}\right)-\zeta\right]^{-1 / 2} d \zeta}{\left[F_{1 / 2}(\nu)-F_{1 / 2}(\nu-\zeta)\right]^{1 / 2}} .
$$

The energy distribution $g(\epsilon)$ is then written as

$$
g(\epsilon)=f(\epsilon) D(\epsilon), \quad f(\epsilon)=\frac{f_{0}}{e^{\alpha+\beta \epsilon}+1} .
$$

The energy distribution is exhibited in Fig. 1 for $r=0.1,1,3$, and 10 . The theoretical curves do not agree with the numerically obtained ones except for the case $r=1$. We note, for $r$ $=1$, that the initial state is near from the virial equilibrium where the virial ratio is unity, and that the energy distribution in QSS is almost the same as the initial one. The Lynden-Bell distribution for the whole system is therefore good when the initial waterbag distribution is near the distribution in the virial equilibrium.

We remark on the simplification of the energy constraint (20b) by using the virial theorem. The virial theorem, in the sheet model, states that the time average of potential energy is equal to twice of the time average of kinetic energy. The Lynden-Bell distribution is a stationary solution to the Vlasov equation, and hence we may replace the time averages with ensemble averages over the distribution. The replacement simplifies the energy constraint (20b) as

$$
U=\frac{3}{\beta}\left(\frac{f_{0}^{2}}{2 \beta^{3}}\right)^{1 / 4} I_{K}(\nu)
$$

and the simplified constraint is used in Refs. [4,5]. The simplified constraint (31) gives almost the same values of $\alpha$ and $\beta$ with ones computed from the original constraint (20b) as shown in Fig. 2. In the next section we will investigate a core distribution, and the virial theorem cannot be expected for the core only. We will therefore adopt the original constraint (20b) and modify it.

\section{DISTRIBUTION IN CORE}

We define core $C$ which is a set of sheets whose energy is less than the threshold $\epsilon_{\mathrm{th}}$, i.e.,

$$
C=\left\{j \text { th sheet } \mid \epsilon_{j}=p_{j}^{2} / 2+\Phi\left(x_{j}\right)<\epsilon_{\mathrm{th}}\right\} .
$$

The complement of core is called halo. The number of elements of $C$ is denoted by $N_{\text {core }}$. We introduce the ratio of core sheets to the all as $R=N_{\text {core }} / N$, and the core energy $U_{\text {core }}$ which is defined as

$$
U_{\text {core }}=\frac{1}{N} \sum_{j \in C}\left[\frac{p_{j}^{2}}{2}+\frac{1}{2 N} \sum_{k=1}^{N}\left|x_{j}-x_{k}\right|\right] .
$$

The factor $1 / 2$ is multiplied in the potential part to equally divide interaction energy into core and halo. The core-halo structure in QSS is shown in Fig. 3 with initial waterbag distribution, where the values of $\epsilon_{\mathrm{th}}$ are chosen from phase portraits on $\mu$ space at QSSs. In Figs. 3(e) and 3(g), the number of sheets belonging to the halo at QSS is asymmetric with respect to the half rotation around the origin, but this asymmetry depends on initial conditions.
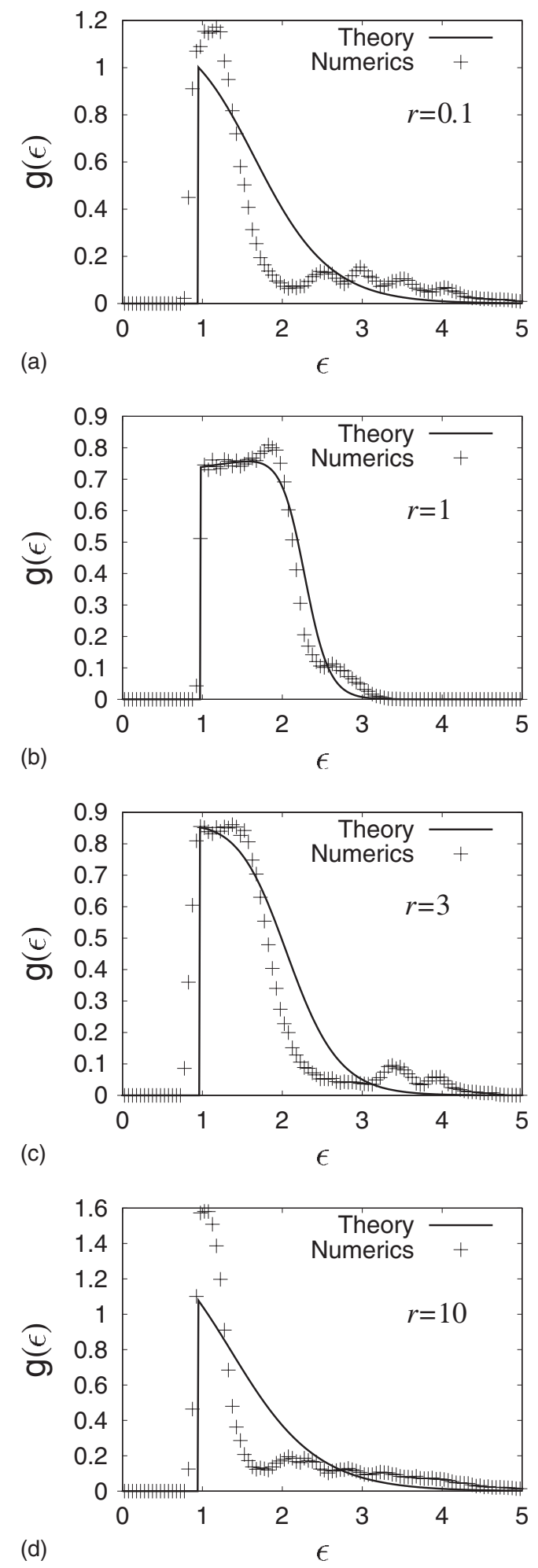

FIG. 1. Energy distribution for $r=0.1$ (a), 1 (b), 3 (c), and 10 (d). In each panel, the solid curve represents the Lynden-Bell distribution and points averaged numerical distributions at $10^{7}$ collisions over 100 realizations. $N=10^{3}$.

Core and halo distributions, which are denoted by $f^{\text {core }}$ and $f^{\text {halo }}$, are introduced by dividing the whole distribution $f$ as 


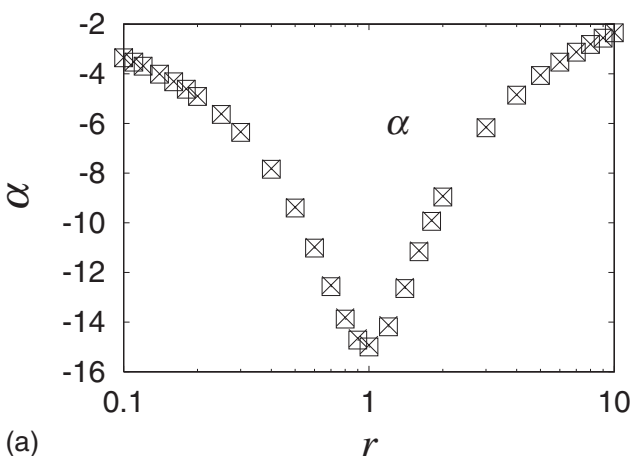

(a)

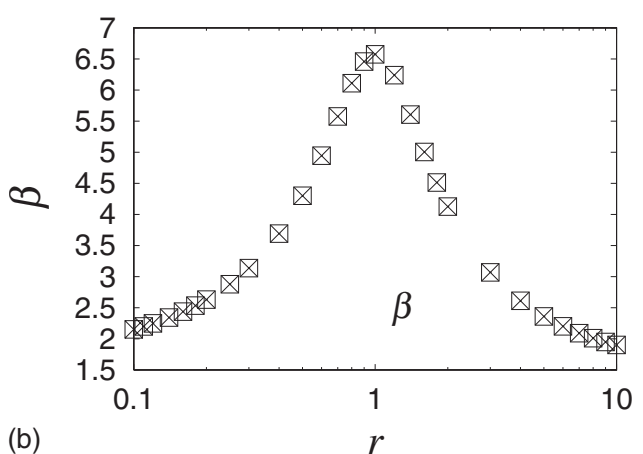

FIG. 2. Lagrange multipliers $\alpha$ (a) and $\beta$ (b) as functions of initial virial ratio $r$ (log scale). Symbols of cross $(\times)$ and square $(\square)$ represent values computed by Eqs. (20b) and (31), respectively.

$$
f=f^{\text {core }}+f^{\text {halo }} .
$$

We will derive a stationary core distribution $f^{\text {core }}(x, p)$ by modifying the two constraints (8a) and (8b).

(1) Normalization. Integration of $f^{\text {core }}$ over $\mu$ space must be the same with the ratio of core sheets to the all, $R$. We hence modify Eq. (8a) as

$$
R=\iint_{\mathbb{R}^{2}} f^{\text {core }}(x, p) d x d p .
$$

(2) Energy constraint. The core energy (33) in the discrete system is associated with

$$
U_{\text {core }}=\iint_{\mathbb{R}^{2}}\left(\frac{p^{2}}{2}+\frac{\Phi[f](x)}{2}\right) f^{\text {core }}(x, p) d x d p
$$

in the continuous system.

Sheets which belong to the halo in the QSS are initially almost confined around the edges of rectangle $D$ (see Fig. 3), and hence initial density $f_{0}$ for core is not modified. The core energy constraint (36) includes the halo distribution through the whole distribution $f$, and hence we must approximate $U_{\text {core }}$ to close the variational problem for the core distribution.

The one-body potential is linear with respect to the distribution, and hence it is also divided into core and halo parts as

$$
\Phi[f](x)=\Phi\left[f^{\text {core }}\right](x)+\Phi\left[f^{\text {halo }}\right](x) .
$$

The modified energy constraint (36) is hence written as

$$
U_{\text {core }}=\iint_{\mathbb{R}^{2}}\left(\frac{p^{2}}{2}+\frac{\Phi\left[f^{\text {core }}\right](x)}{2}\right) f^{\text {core }}(x, p) d x d p+V_{\text {core-halo }}
$$

where the second term of the right-hand side

$$
\begin{aligned}
V_{\text {core-halo }}= & \frac{1}{2} \iint_{\mathbb{R}^{2}} \Phi\left[f^{\text {halo }}\right](x) f^{\text {core }}(x, p) d x d p \\
= & \frac{1}{2} \iint_{\mathbb{R}^{2}} d x d p \iint_{\mathbb{R}^{2}}|x-y| f^{\text {halo }}\left(y, p^{\prime}\right) \\
& \times f^{\text {core }}(x, p) d y d p^{\prime}
\end{aligned}
$$

represents gravitational interactions between core and halo. To eliminate the halo distribution $f^{\text {halo }}$ from the potential term $V_{\text {core-halo, }}$, we approximate that the position of halo sheet $y$ is greater than the position of core sheet $x$ for $y>0$ and $y<x$ for $y<0$. This approximation and the symmetry $f^{\text {halo }}\left(-y, p^{\prime}\right)=f^{\text {halo }}\left(y, p^{\prime}\right)$ lead to

$$
\begin{aligned}
V_{\text {core-halo }} & =\frac{1}{2} \iint_{\mathbb{R}^{2}} f^{\text {core }}(x, p) d x d p \int_{\mathbb{R}} d p^{\prime} \int_{0}^{\infty} 2 y f^{\text {halo }}\left(y, p^{\prime}\right) d y \\
& =\frac{1}{2} R \iint_{\mathbb{R}^{2}}|y| f^{\text {halo }}\left(y, p^{\prime}\right) d y d p^{\prime}=R \frac{\phi_{0}^{\text {halo }}}{2},
\end{aligned}
$$

where $\phi_{0}^{\text {halo }}=\Phi\left[f^{\text {halo }}\right](0)$. To this end, the modified energy constraint (38) is approximated as

$$
U_{\text {core }}=\iint_{\mathbb{R}^{2}}\left(\frac{p^{2}}{2}+\frac{\Phi\left[f^{\text {core }}\right](x)}{2}\right) f^{\text {core }}(x, p) d x d p+R \frac{\phi_{0}^{\text {halo }}}{2}
$$

and $f^{\text {halo }}$ has been eliminated.

By using two Lagrange multipliers $\alpha_{\text {core }}$ and $\beta_{\text {core }}$ associated to the two constraints (35) and (41), respectively, the core distribution is written as

$$
f^{\text {core }}(x, p)=\frac{f_{0}}{e^{\alpha_{\text {core }}+\beta_{\text {core }}\left\{p^{2} / 2+\Phi\left[f^{\text {core }}\right](x)+\phi_{0}^{\text {halo }}\right\}}+1} .
$$

The term $\beta_{\text {core }} \phi_{0}^{\text {halo }}$ in the denominator of the right-hand side is extracted from $\alpha_{\text {core }}$ for adjusting the zero-point potential energy to $\phi_{0}$. The two Lagrange multipliers are determined by the two modified constraints

$$
\begin{aligned}
& R=4\left(\frac{f_{0}^{2}}{2 \beta_{\text {core }}^{3}}\right)^{1 / 4}\left[F_{1 / 2}\left(\nu_{\text {core }}\right)\right]^{1 / 2}, \\
& U_{\text {core }}= \frac{1}{2 \beta_{\text {core }}}\left(\frac{f_{0}^{2}}{2 \beta_{\text {core }}^{3}}\right)^{1 / 4}\left[2 I_{K}\left(\nu_{\text {core }}\right)+I_{V}\left(\nu_{\text {core }}\right)\right] \\
&+\frac{R}{2}\left(\frac{1}{\beta_{\text {core }}} I_{\phi}\left(\nu_{\text {core }}\right)+\phi_{0}^{\text {halo }}\right),
\end{aligned}
$$

where $\nu_{\text {core }}=-\alpha_{\text {core }}-\beta_{\text {core }} \phi_{0}, \phi_{0}=\phi_{0}^{\text {core }}+\phi_{0}^{\text {halo }}$, and 

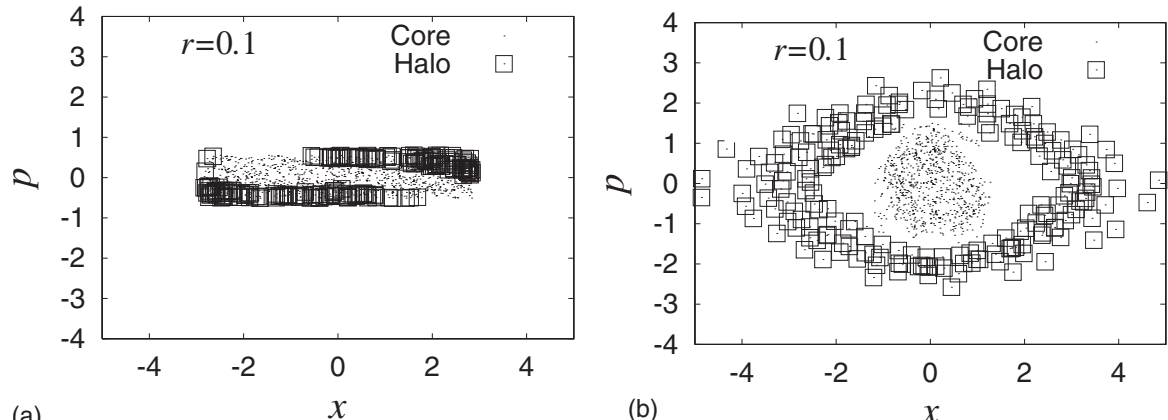

(a)

(b)
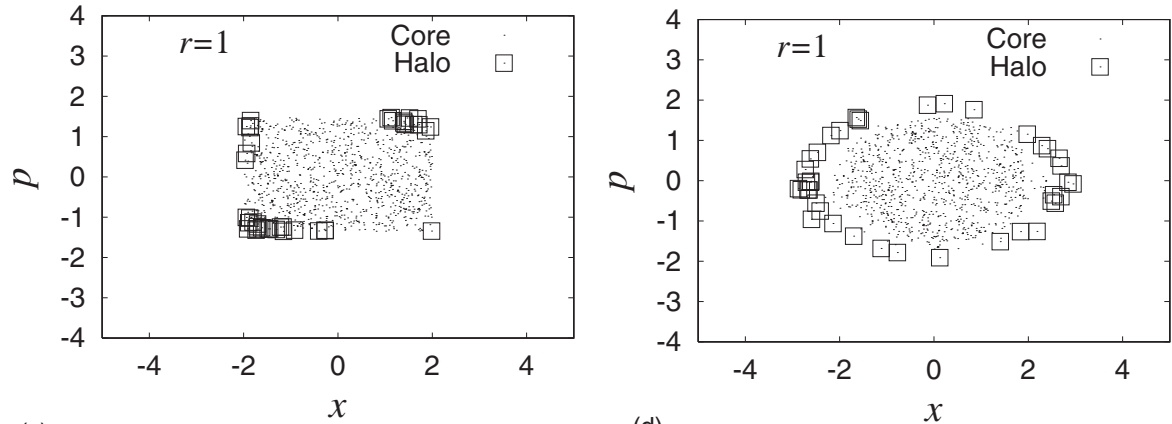

(c)

(d)
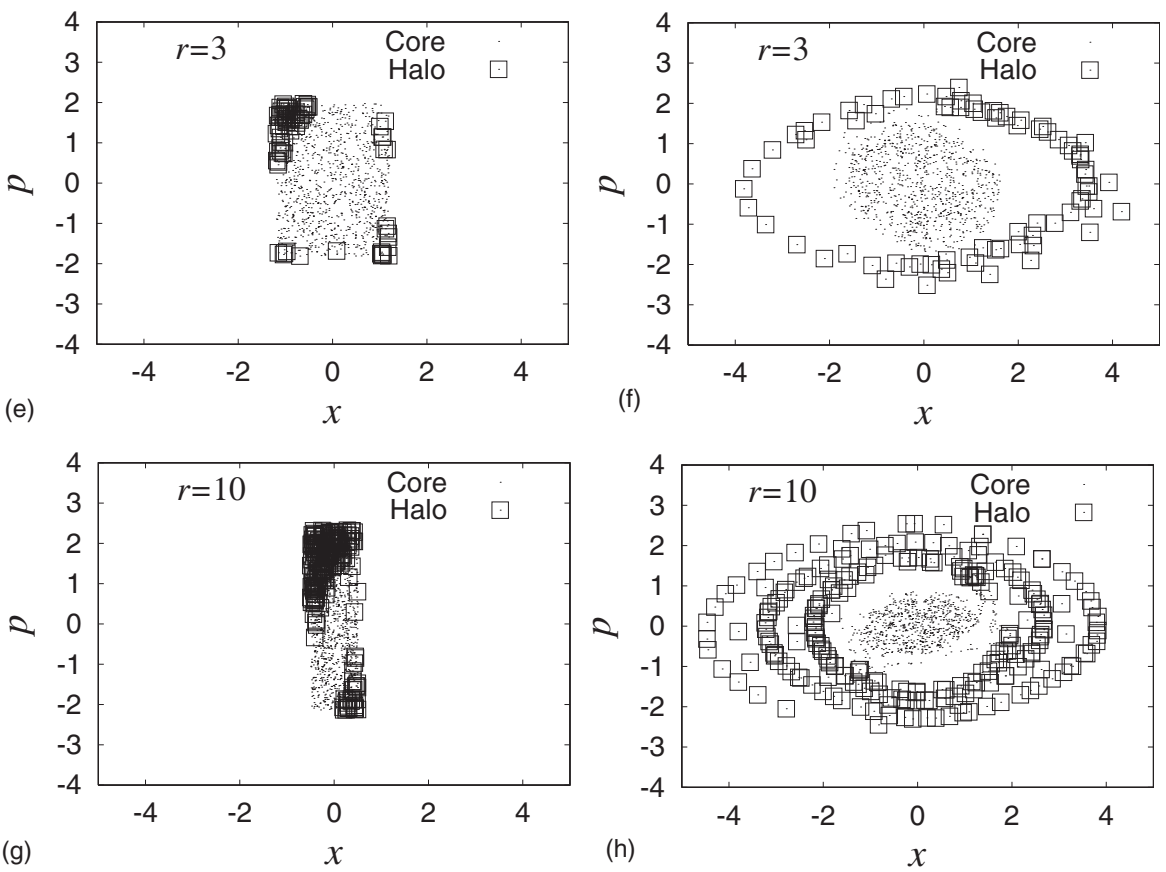

FIG. 3. Initial states (a),(c),(e),(g), and QSSs after $10^{7}$ collisions (b),(d),(f),(h) on $\mu$ space. The initial virial ratio is $r=0.1$ [(a),(b)], 1 $[(\mathrm{c}),(\mathrm{d})], 3[(\mathrm{e}),(\mathrm{f})]$, and $10[(\mathrm{~g}),(\mathrm{h})]$, and the threshold energy is chosen as $\epsilon_{\mathrm{th}}=2.2,2.6,2.6$, and 2.0, respectively. Big square and dot represent a sheet belonging to halo and to core after $10^{7}$ collisions, respectively. $N=10^{3}$.

$$
\phi_{0}^{\text {core }}=\Phi\left[f^{\text {core }}\right](0)=\frac{1}{\beta_{\text {core }}} I_{\phi}\left(\nu_{\text {core }}\right) .
$$

The multiplier $\beta_{\text {core }}$ is solved as

$$
\beta_{\text {core }}=\left\{\frac{2^{7}\left[f_{0} F_{1 / 2}\left(\nu_{\text {core }}\right)\right]^{2}}{R^{4}}\right\}^{1 / 3}
$$

by the modified normalization condition (43a) and the value of $\nu_{\text {core }}$ is computed as a solution to Eq. (43b).
According to the core distribution (42), one particle energy in core can be defined as

$$
\epsilon=\frac{p^{2}}{2}+\Phi\left[f^{\text {core }}\right](x)+\phi_{0}^{\text {halo }}
$$

The energy distribution in the core $g^{\text {core }}(\epsilon)$ is hence expressed as 
TABLE I. Parameters for the core part. $N=10^{3}$ and $U=1$. Values of $U_{\text {core }}$ and $R$ are averages over 100 sample orbits.

\begin{tabular}{lccc}
\hline \hline$r$ & $\epsilon_{\text {th }}$ & $U_{\text {core }}$ & $R$ \\
\hline 0.1 & 2.2 & 0.5833 & 0.7986 \\
1 & 2.6 & 0.9315 & 0.9628 \\
3 & 2.6 & 0.7948 & 0.9118 \\
10 & 2.0 & 0.4831 & 0.7362 \\
\hline \hline
\end{tabular}

$$
g^{\text {core }}(\epsilon)=f^{\text {core }}(\epsilon) D_{\text {core }}(\epsilon),
$$

where

$$
f^{\text {core }}(\epsilon)=\frac{f_{0}}{e^{\alpha_{\text {core }}+\beta_{\text {core }} \epsilon}+1}
$$

and

$$
\begin{aligned}
D_{\text {core }}(\epsilon)= & \left(\frac{\beta_{\text {core }}}{2 f_{0}^{2}}\right)^{1 / 4} \\
& \times \int_{0}^{\beta_{\text {core }}\left(\epsilon-\phi_{0}\right)} \frac{\left[\beta_{\text {core }}\left(\epsilon-\phi_{0}\right)-\zeta\right]^{-1 / 2} d \zeta}{\left[F_{1 / 2}\left(\nu_{\text {core }}\right)-F_{1 / 2}\left(\nu_{\text {core }}-\zeta\right)\right]^{1 / 2}} .
\end{aligned}
$$

We shortly summarize the procedure to compute the core energy distribution. The value of $\phi_{0}$ is obtained from the procedure to compute the whole distribution, shown in Sec. IV. A solution to Eq. (43b) gives a value of $\nu_{\text {core }}$ and $\nu_{\text {core }}$ gives $\beta_{\text {core }}$ by Eq. (45) and $\phi_{0}^{\text {core }}$ by Eq. (44). The other Lagrange multiplier $\alpha_{\text {core }}$ is computed by the relation $\alpha_{\text {core }}$ $=-\nu_{\text {core }}-\beta_{\text {core }} \phi_{0}$. Finally $\phi_{0}^{\text {halo }}$ is obtained by the relation $\phi_{0}^{\text {halo }}=\phi_{0}-\phi_{0}^{\text {core }}$.

Two parameters $R$ and $U_{\text {core, }}$ introduced in the two modified constraints, are not determined in this procedure. We hence compute their values by numerical $N$-body simulations, and the values are arranged in Table I.

In the computation of core distribution, we used the value of $\phi_{0}$, which is obtained from computations of the whole distribution. The whole distribution does not agree with numerical simulations, but the value of $\phi_{0}$ agrees as shown in Fig. 4. The discrepancy between the theoretical curve and the numerical one is up to 0.06 in the computed interval of $r$, which is not large compared to the fixed specific energy $U$ $=1$.

The values of $\phi_{0}$ is also observed in Fig. 1 as energy where the energy distribution starts to increase. Distributions in QSSs are symmetric with respect to the change in sign of $x$ (see Fig. 3), and hence $\Phi[f](x)$ is an increasing function in $x>0$ as shown in Sec. IV. Accordingly,

$$
\epsilon=\frac{p^{2}}{2}+\Phi[f](x) \geqslant \Phi[f](0)=\phi_{0}
$$

and hence $g(\epsilon)=0$ for $\epsilon<\phi_{0}$. In Fig. 1, the starting energy $\phi_{0}$ of the theoretical curve is in good agreement with numerical one. We remark that the minimum value is also $\phi_{0}$ for oneparticle energy in the core, Eq. (46), thanks to the extracted term $\phi_{0}^{\text {halo }}$ in the core distribution (42).

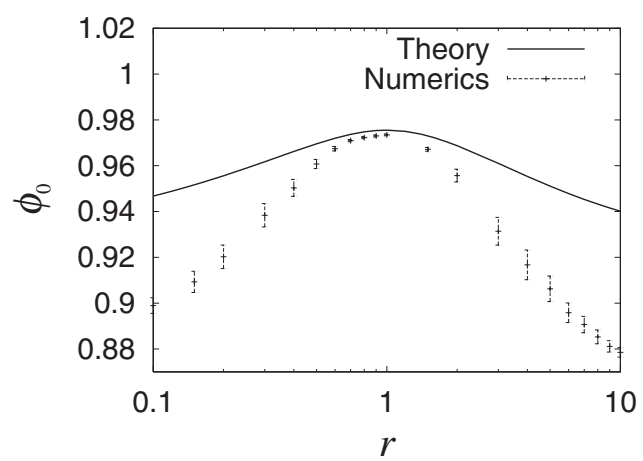

FIG. 4. The zero-point potential energy $\phi_{0}$ as a function of the initial virial ratio $r(\log$ scale). Solid line represents the theoretical curve. Points and error bars represent averages and standard deviations over the samples which are taken in the interval from $5.10^{6}$ to $10^{7}$ collisions for 100 realizations. $N=10^{3}$.

To this end, the core energy distribution $g^{\operatorname{core}}(\epsilon)$ is compared with the numerically obtained one in Fig. 5. The theoretical distribution agrees with numerical one in the expected energy interval $\phi_{0}<\epsilon \lesssim \epsilon_{\mathrm{th}}$. This agreement suggests (i) that the core is formed by a statistical mechanism rather than the dynamical mechanism which generates the halo and (ii) that the basic idea of exclusivity is valid to derive distributions in QSSs if we focus on the core only.

Finally we mention the relation between $U_{\text {core }}$ and $R$. A fixed value of the threshold $\epsilon_{\mathrm{th}}$ determines a pair of $\left(U_{\text {core }}, R\right)$, and we compute many pairs of $\left(U_{\text {core }}, R\right)$ by changing the value of $\epsilon_{\mathrm{th}}$. The pairs are pointed on the twodimensional $R-U_{\text {core }}$ plane in Fig. 6, and they are approximately on a line. The least square method for $R>0.7$ gives the relation $U_{\text {core }}=1.84 R-0.86$, and the relation is reasonable since $U_{\text {core }}$ is near 1 for $R=1$, that is, all the sheets are in core. This relation implies that we can compute $U_{\text {core }}$ and can predict the core distribution by counting the numbers of sheets in the core and halo.

\section{SUMMARY AND DISCUSSIONS}

We studied the sheet model to examine validity of the Lynden-Bell statistics. The Lynden-Bell distribution is not suitable to fit the whole distribution when initial virial ratio $r$ is much smaller or much larger than 1 , where $r=1$ implies that the initial distribution is near the virial equilibrium. In the sheet model sheets are classified into core and halo. Halo is dynamically constructed, and hence its distribution cannot be predicted by a statistical treatment. However, a modified Lynden-Bell statistics describes the core distribution and hence the essence of the Lynden-Bell statistics is still effective even in the sheet model.

To construct the core energy distribution, we required two parameters, which are core energy $U_{\text {core }}$ and the number ratio $R$ for a fixed value of threshold energy $\epsilon_{\mathrm{th}}$. Thanks to a linear relation between the two parameters, we can reduce the number of unknown parameters to 1 . However, the value of the parameter is not determined from a given initial condition, and hence the procedure proposed in this paper is not selfcontained. Qualitative analysis on the dynamical construc- 


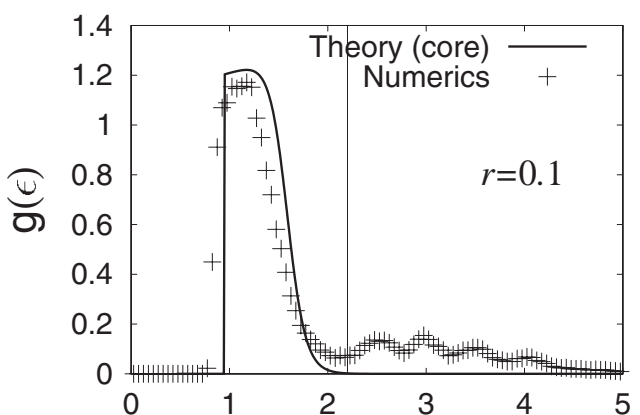

(a)

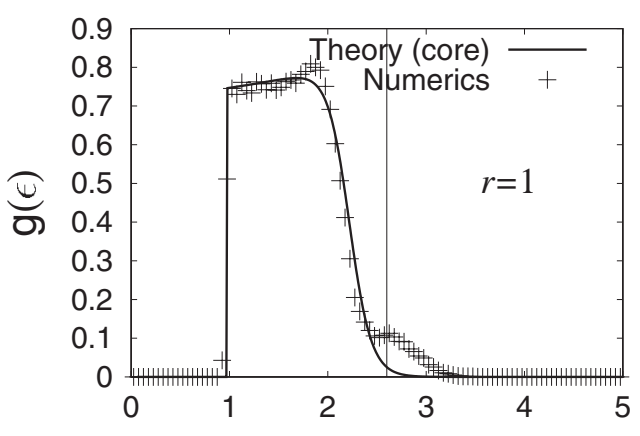

(b)

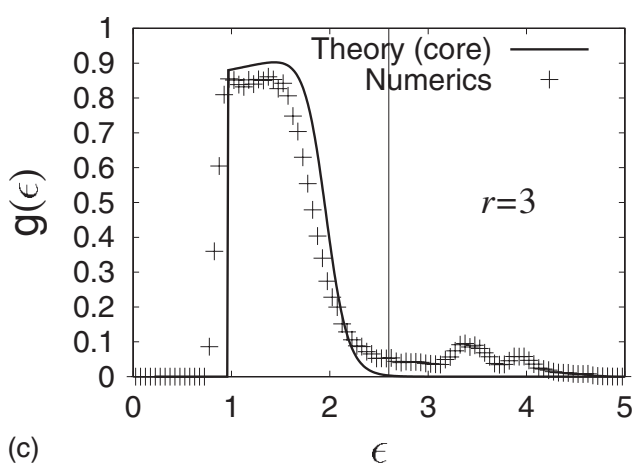

(c)

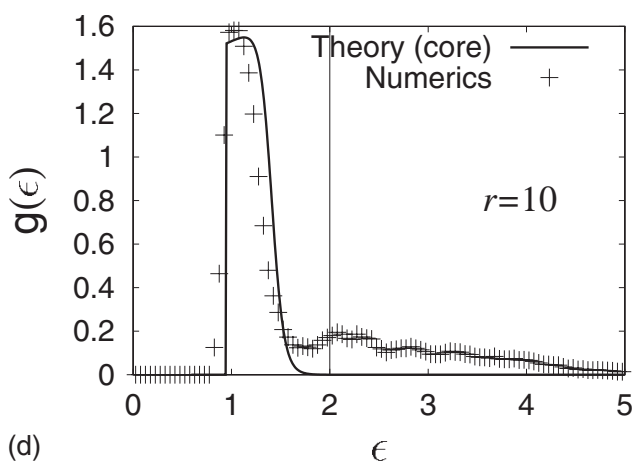

FIG. 5. Energy distribution for $r=0.1$ (a), 1 (b), 3 (c), and 10 (d). In each panel, the solid curve represents the theoretical core distribution and points numerical distributions which are the same with ones reported in Fig. 1. The vertical lines mark the threshold $\epsilon_{\mathrm{th}} \cdot N=10^{3}$.

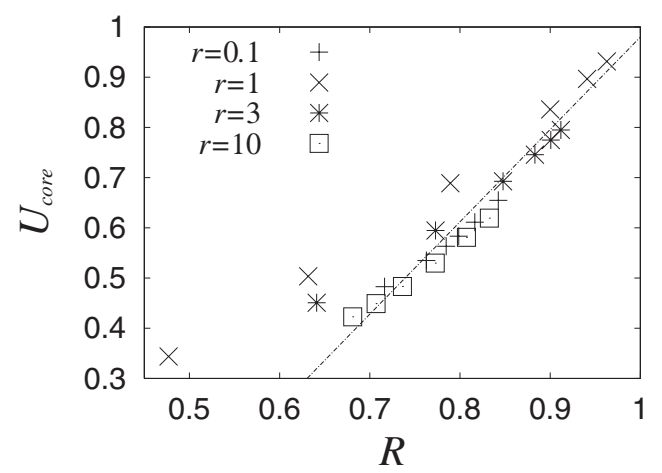

FIG. 6. Relation between $U_{\text {core }}$ (vertical axis) and $R$ (horizontal axis) for various values of $\epsilon_{\mathrm{th}}$ and $r=0.1,1,3,10$. The line is obtained by the least square method for $R>0.7$, and represents $U_{\text {core }}$ $=1.84 R-0.86 . N=10^{3}$.

tion of the halo has been discussed [4], and the quantitative analysis may determine the value of the parameter.

The value of threshold energy $\epsilon_{\mathrm{th}}$ is chosen from phase portraits in $\mu$ space. The threshold energy is hence a free parameter, but theoretical core energy distributions are similar if the values of $\epsilon_{\mathrm{th}}$ are close. The abovementioned quantitative analysis may also give a systematic criterion to determine the value of $\epsilon_{\mathrm{th}}$.

In Fig. 5(d), the numerical distribution increases around energy threshold $\epsilon_{\mathrm{th}}=2.0$, while the theoretical curve monotonically decreases. This increase is produced by the edge of halo, since halo is not perfectly disconnected from the core. To remove the unexpected increase of distribution in the core energy region, one can redefine the value of threshold energy, for instance, $\epsilon_{\mathrm{th}}=1.9$, but no solution could be found to solve constraint equations in the range of computations. This failure may come from numerical difficulties to handle Fermi degeneracy in the core, in other words, divergence of $\beta_{\text {core }}$.

The statistical mechanics introduced in this paper is based on the approximation of the discrete $N$-body system by the continuous Vlasov equation. The approximation is verified by a theorem due to Braun and Hepp [23]. The theorem requires that the potential function expressing the two-body interaction is smooth enough, but it is not smooth at collision in the sheet model. It is up to future work to study whether the nonsmoothness prevents us from constructing the statistical mechanics for the whole system. The theorem also requires one to take the limit $N \rightarrow \infty$, and $N=1000$ performed in this paper is possibly small. Performing numerical computations with larger $N$ is another future work.

\section{ACKNOWLEDGMENTS}

The author thanks Atsushi Taruya for valuable discussions and comments. This work has been supported by the Ministry of Educations, Science, Sports and Culture, Grant-in-Aid for Young Scientists (B), Grant No. 19760052, 2008. 
[1] D. Lynden-Bell, Mon. Not. R. Astron. Soc. 136, 101 (1967).

[2] F. Hohl, NASA Technical Report No. NASA TR R-289 (1968).

[3] F. Hohl and J. W. Campbell, NASA Technical Report No. NASA TN D-5540 (1969).

[4] T. Yamashiro, N. Gouda, and M. Sakagami, Prog. Theor. Phys. 88, 269 (1992).

[5] M. Lecar and L. Cohen, Astrophys. Space Sci. 13, 397 (1971).

[6] P. Mineau, M. R. Feix, and J. L. Rouet, Astron. Astrophys. 228, 344 (1990).

[7] T. Tsuchiya, T. Konishi, and N. Gouda, Phys. Rev. E 50, 2607 (1994).

[8] J. Barré, T. Dauxois, G. De Ninno, D. Fanelli, and S. Ruffo, Phys. Rev. E 69, 045501(R) (2004).

[9] M. Antoni and S. Ruffo, Phys. Rev. E 52, 2361 (1995).

[10] V. Pavlov, D. Buisine, and S. Decossin, Phys. Fluids 14, 3937 (2002).

[11] R. Kawahara and H. Nakanishi, J. Phys. Soc. Jpn. 75, 054001 (2006).

[12] X.-P. Huang and C. F. Driscoll, Phys. Rev. Lett. 72, 2187 (1994).
[13] K. S. Fine, A. C. Cass, W. G. Flynn, and C. F. Driscoll, Phys. Rev. Lett. 75, 3277 (1995).

[14] Y. Kiwamoto, N. Hashizume, Y. Soga, J. Aoki, and Y. Kawai, Phys. Rev. Lett. 99, 115002 (2007).

[15] Y. Y. Yamaguchi, J. Barré, F. Bouchet, T. Dauxois, and S. Ruffo, Physica A 337, 36 (2004).

[16] J. Barré, F. Bouchet, T. Dauxois, S. Ruffo, and Y. Y. Yamaguchi, Physica A 365, 177 (2006).

[17] A. Antoniazzi, D. Fanelli, J. Barré, P.-H. Chavanis, T. Dauxois, and S. Ruffo, Phys. Rev. E 75, 011112 (2007).

[18] A. Antoniazzi, D. Fanelli, S. Ruffo, and Y. Y. Yamaguchi, Phys. Rev. Lett. 99, 040601 (2007).

[19] J. Miller, Phys. Rev. Lett. 65, 2137 (1990).

[20] J. Miller, P. B. Weichman, and M. C. Cross, Phys. Rev. A 45, 2328 (1992).

[21] D. Z. Jin and D. H. E. Dubin, Phys. Rev. Lett. 80, 4434 (1998).

[22] P.-H. Chavanis, J. Sommeria, and R. Robert, Astrophys. J. 471, 385 (1996).

[23] W. Braun and H. Hepp, Commun. Math. Phys. 56, 101 (1977). 